\title{
Kinematic Simulation and Analysis of Globoidal Indexing Cam
}

\author{
Hu Xinzhe ${ }^{1,}$, Feng Huize ${ }^{1}$, Tang Huiying ${ }^{1}$, Hong Xueming ${ }^{1}$, Fan Jiankai ${ }^{2}$ \\ ${ }^{1}$ Tianjin BENEFO Mechanical Equipment Central Institute, Mechanical Laboratory, 300350, Tianjin, China \\ ${ }^{2}$ Beijing Institute of Space Mechanics \& Electricity, Mechanism Department, Beijing, 100000, China
}

\begin{abstract}
As an important mechanism with intermittent motion, the globoidal indexing cam is always a research hot in the mechanical fields. The working profile of globoidal indexing cam is extremely complicated and undevelopable, which make it quite difficult to be protracted by the conventional drafting method. Aiming at this problem, the working curvilinear equation of the intermittent motion of an indexing cam is derived based on the RPY (Roll-Pitch-Yaw) coordinate transformation method. The 3D model based on the curvilinear equation is built by the Creo2.0 modeling software. The virtual prototype is established based on the ADAMS software, while the kinematics simulation is implemented. The success of virtual simulation verifies the correctness of curvilinear equation. The numerical results, presented and discussed in the paper, indicate that the proposed model is feasible to foresee the kinematic behaviour of an actual system.
\end{abstract}

\section{Introduction}

As an important branch of the mechanism fields, indexing mechanisms have been developed rapidly in recent decades. In engineering, the globoidal indexing cam(GIC), with its guide and control functions, is a kind of important and potential mechanism, widely employed to drive rotary platforms, convey belts and automatic tool changers(ATC) in machine tools, and also applied in light manufacturing, auto parts, machine manufacturing, and transport equipment, etc $[1,2]$. Figure 1 . shows the example of industrial applications which employing a GIC.

(a)

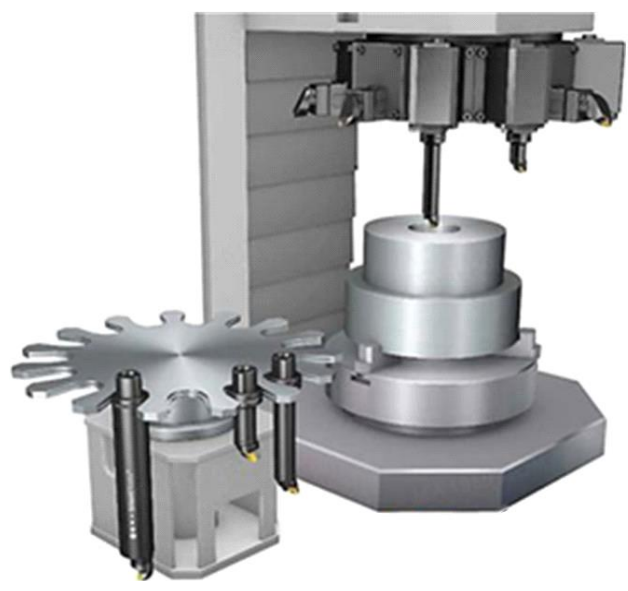

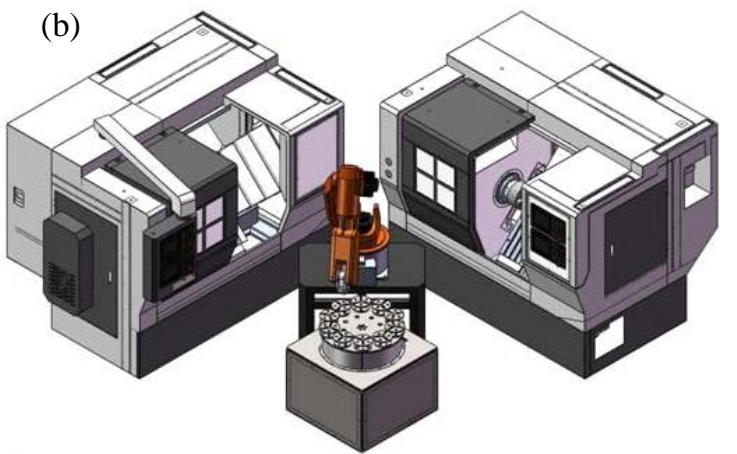

Figure 1. Examples of industrial applications employing a GIC: (a) ATC in machine tool; (b) rotary platform for robot

The GIC is developed by Neklutin C. N. in 1920s, who was an American [3]. Other countries like the former Soviet Union, Switzerland, England, German, and Japan are going to research this mechanism systematically [4-6], their research focus are mostly related to the design, manufacture and inspection, etc. The GIC has the advantages as follows [7]: (1) Simple structure, high stiffness, small axial dimensions, highest bearing capacity in cam mechanism. (2) High speed, large indexing numbers, high indexing precision. (3) The prestressing force can be applied on the drive and driven shaft by adjusting the clearance between the indexing cam and dial, which can guarantee the well dynamic and kinetic characteristics and smooth operation. The GIC has a unique and superior role in the high precision transmission and intermittent movement $[8,9]$, and the motion law design directly influences the operation accuracy of the cam mechanism [10], so the working profile and kinematics has become an important research 
subject [11]. The obstacle, however, is the lack of effective methods to obtain the precise working profile.

Based on the RPY coordinate transformation method, this paper derives the equation of working curvilinear. The virtual prototype of the indexing cam by using the Creo modeling software is presented and the kinematics simulation is implemented based on the ADAMS software.

\section{Prototype mechanism of globoidal indexing cam layout}

\subsection{The structure analysis of globoidal indexing cam}

As shown in Figure 2, GIC mechanical is an indexing mechanism with high speed and high accuracy for intermittent transmission, which is composed of a concave cam (the under one) and a dial with several radially installed rollers (the upper one). The intermittent motion (dwell-run-dwell) is generated by the particular shape of the cam.

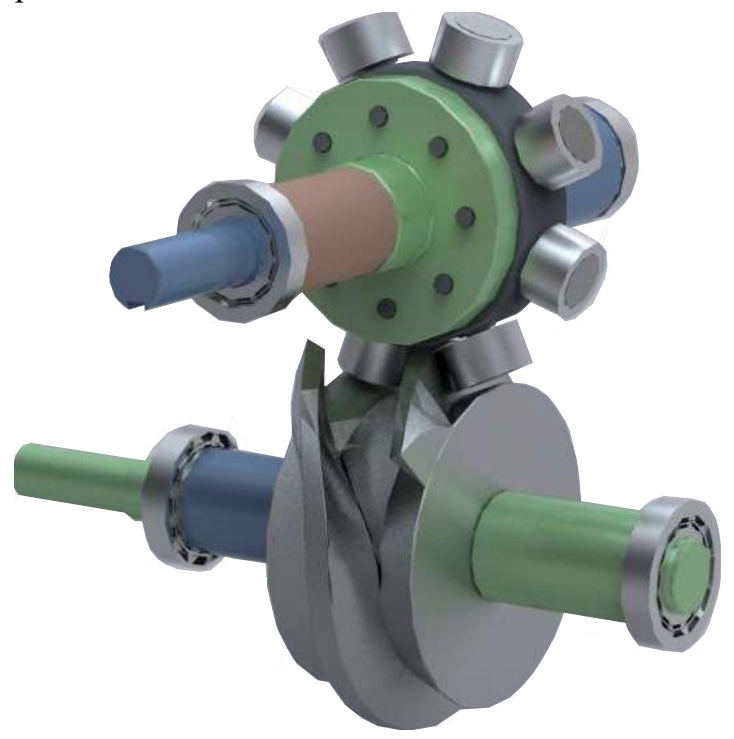

Figure 2. The assemblies of globoidal indexing cam

During the indexing phase, the spatial cam with ridges propels rollers rotate slowly with the cam. In the stopping phase, the adjacent rollers in the dial cross on the cam ridges and the dial stop spinning, which will guarantee the stability of the mechanism. Beside the orientating function, the more important function of the GIC is that the mechanism can eliminate the clearance automatically and compensate the abrasion between the cam and dial without any auxiliary devices.

However, the indexing cam is one of the core components in the power unit of the GIC, and the complicated shape of globoidal cam profile and nondevelopable surface, making it more difficult to design and machine it. So its manufacture quality requirement is very high.

\subsection{Establishment of indexing cam working curvilinear equation}

In order to facilitate the analysis and design, as Figure 3. shows, the stationary Cartesian coordinate systems $O_{1}$ $X_{1} Y_{1} Z_{1}$ (denoted as $\left\{F_{1}\right\}$ ) is set up with its origin $O_{1}$ fixed in the rank of rotational center on the indexing cam, $X_{1}$ axis has the same direction with $O_{2} O_{1}\left(O_{2}\right.$ is just the rotation center of the dial). The direction of $Z_{1}$ axis conform with the right-hand screw rule, in other words, hold the four fingers straight with the end face of indexing cam and point to the rotation direction of indexing cam, and then close them against the thumb, now the thumb will show the positive direction of the $Z_{1}$ axis. The origin of the other stationary coordinate systems $O_{2}-X_{2} Y_{2} Z_{2}$ (denoted as $\left\{F_{2}\right\}$ ) is fixed in the rank of rotational center on the dial, $X_{2}$ is along the same direction with $\mathrm{O}_{2} \mathrm{O}_{1}$, and $Y_{2}$ has the same direction with $Z_{1}$.

The moving coordinate system $o_{1}-x_{1} y_{1} z_{1}$ (denoted as $\left.\left\{M_{1}\right\}\right)$ is fixed on the rotational center of the indexing cam, whose initial orientation coincides with that of the frame $\left\{F_{1}\right\}$. The moving coordinate system $o_{2}-x_{2} y_{2} z_{2}$ is fixed on the rotational center of the dial, whose roller is numbered by No.1 to No.8, the $x_{2}$ axis is coincides with the axis of No. 2 roller, $z_{2}$ has the same direction with $Z_{2}$, and the angle between $x_{2}$ and the positive $X_{2}$ is $\phi$. The frame $\left\{M_{1}\right\}$ rotates with the rotation of the indexing cam, so does the $\left\{M_{2}\right\}$ with dial. When the rotation angle of indexing cam is $\theta$, the angle between $X_{1}$ and $x_{1}$ is also $\theta$, which is consistent with the cam rotation angle.

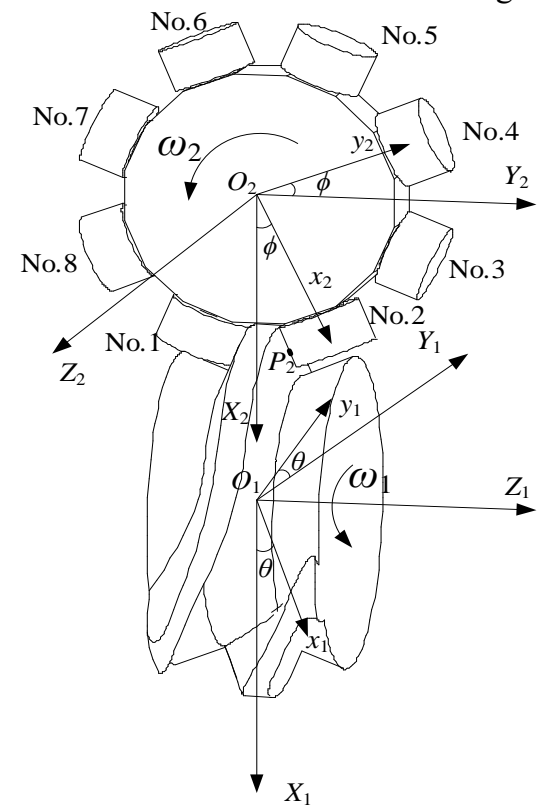

Figure 3. Globoidal indexing cam mechanism

As shown in Figure 4, the cross section of No.2 roller is projected onto the plane $y_{2} O_{2} z_{2}$ for conveniently describing the equation of the work-point $P_{2}$, and whose projection point in the $y_{2} O_{2} z_{2}$ is represented by $P_{2}^{\prime}$.

The coordinate vector of $P_{2}$ in the stationary $\left\{M_{2}\right\}$ coordinate system can be expressed as

$$
r_{P_{2}}=\left[\begin{array}{lll}
r & \rho \cos \psi & \rho \sin \psi
\end{array}\right]^{T}
$$


Where, $r$ represents the $x_{2}$-value of the work-point, $\rho$ represents the radius of roller, and $\psi$ represents the angle between $O_{2} P_{2}^{\prime}$ and the positive $y_{2}$-axis.

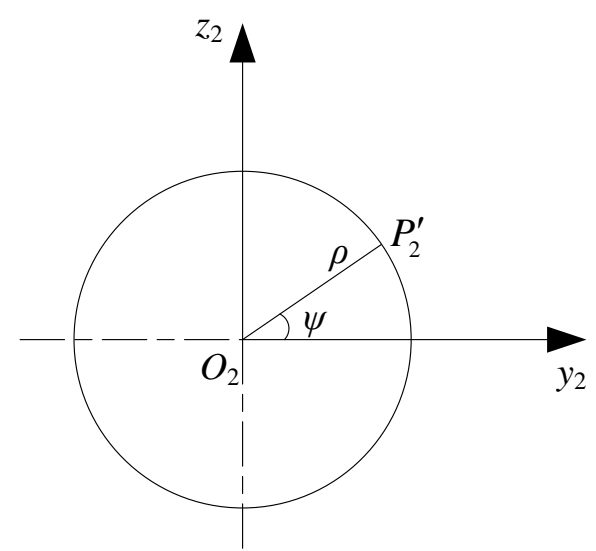

Figure 4. The cross section projection of No.2 roller

In the stationary coordinate systems $\left\{F_{2}\right\}$, the workpoint $P_{2}$ can be expressed as

$$
P_{2}=R\left(Z_{2}, \phi\right) r_{P_{2}}
$$

Where, $R\left(Z_{2}, \phi\right)$ is the $\operatorname{RPY}(X-Y-Z)$ transformation matrix from the moving coordinate system $\left\{M_{2}\right\}$ to the stationary coordinate system $\left\{F_{2}\right\}$, which can be expressed as

$$
R\left(Z_{2}, \phi\right)=\left[\begin{array}{ccc}
c \phi & -s \phi & 0 \\
s \phi & c \phi & 0 \\
0 & 0 & 1
\end{array}\right]
$$

Where, $c \phi$ means $\cos \phi, s \phi$ means $\sin \phi$, the rest remains the same.

By substituting Eq. (1) into Eq. (2), the following equation can be obtained:

$$
P_{2}=\left[\begin{array}{c}
r c \phi-\rho c \psi s \phi \\
r s \phi+\rho c \psi c \phi \\
\rho s \psi
\end{array}\right]
$$

Similarly, in the stationary coordinate systems $\left\{F_{1}\right\}$, the work-point $P_{1}$ can be expressed as

$$
P_{1}=R\left(Z_{1}, \theta\right) r_{P 1}
$$

Where, $R\left(Z_{1}, \phi\right)$ is the transformation matrix from the moving coordinate system $\left\{M_{1}\right\}$ to the stationary coordinate system $\left\{F_{1}\right\}, \quad r_{P 1}=\left[\begin{array}{lll}x & y & z\end{array}\right]^{T}$ is the coordinate vector of work-point $P_{1}$ in the moving coordinate system $\left\{M_{1}\right\}$, and $x, y, z$, represents the $x_{1-}$ value, $y_{1}$-value, $z_{1}$-value of the work-point $\mathrm{P} 1$, respectively. So, Eq. (4) can be expressed as

$$
P_{1}=\left[\begin{array}{ccc}
c \theta & -s \theta & 0 \\
s \theta & c \theta & 0 \\
0 & 0 & 1
\end{array}\right]\left[\begin{array}{l}
x \\
y \\
z
\end{array}\right]=\left[\begin{array}{c}
c \theta x-s \theta y \\
s \theta x+c \theta y \\
z
\end{array}\right]
$$

And in the stationary coordinate system $\left\{F_{1}\right\}$, the work-point $P_{2}$ can be expressed as

$$
P_{1}=R\left(X_{1}, 90^{\circ}\right) P_{2}+T
$$

Where, $R\left(X_{1}, 90^{\circ}\right)$ and $T$ are the mapping relationship from $\left\{F_{2}\right\}$ to $\left\{F_{1}\right\}$, they are respectively represented as following

$$
\begin{aligned}
& R\left(X_{1}, 90^{\circ}\right)=\left[\begin{array}{ccc}
1 & 0 & 0 \\
0 & c 90^{\circ} & -s 90^{\circ} \\
0 & s 90^{\circ} & c 90^{\circ}
\end{array}\right]=\left[\begin{array}{ccc}
1 & 0 & 0 \\
0 & 0 & -1 \\
0 & 1 & 0
\end{array}\right], \\
& T=\left[-\left|O_{1} O_{2}\right| \quad 0 \quad 0\right]^{T}=\left[\begin{array}{lll}
-a & 0 & 0
\end{array}\right], \text { and a is the }
\end{aligned}
$$
center distance between indexing cam and dial. By substituting Eq. (3) into Eq. (6), the following equation can be obtained

$$
\begin{aligned}
P_{1} & =\left[\begin{array}{ccc}
1 & 0 & 0 \\
0 & 0 & -1 \\
0 & 1 & 0
\end{array}\right]\left[\begin{array}{c}
r c \phi-\rho c \psi s \phi \\
r s \phi+\rho c \psi c \phi \\
\rho s \psi
\end{array}\right]+\left[\begin{array}{c}
-a \\
0 \\
0
\end{array}\right] \\
& =\left[\begin{array}{c}
r c \phi-\rho c \psi s \phi-a \\
-\rho s \psi \\
r s \phi+\rho c \psi c \phi
\end{array}\right]
\end{aligned}
$$

The working curvilinear equation of the indexing cam is obtained by solving the simultaneous equations, which is composed by Eq. (5) and Eq. (7)

$$
\left\{\begin{array}{l}
x=r c \phi c \theta-\rho c \psi s \phi c \theta-\rho s \psi s \theta-a c \theta \\
y=-r c \phi s \theta+\rho c \psi s \phi s \theta-\rho s \psi c \theta+a s \theta \\
z=r s \phi+\rho c \psi c \phi
\end{array}\right.
$$

\section{The kinematics simulation globoidal indexing cam}

\subsection{The establishment of GIC model}

It's needn't to make the virtual model keep consistent with the detail parts of practical mechanism deliberately; otherwise it will waste too much time on modeling, however, obtaining the reliable simulation results is the crucial point. So in order to obtain the high simulation quality and efficiency, the model should be simplified, and the component number of the mechanism should be reduced before modeling.

The design parameters of GIC are as followings: center distance $a=180 \mathrm{~mm}$, dial radius $R=96 \mathrm{~mm}$, roller radius $R_{r}=22 \mathrm{~mm}$, roller number $n=8 \mathrm{~mm}$, cam angle in indexing phase $\theta_{f}=120^{\circ}$, interval between cam and dial $b=0.1 \mathrm{~mm}$, and the motion law of dial is modified sinusoid arcs.

As shown in figure 5, the cam curves are plotted by the program which are complied by the Creo2.0 language based on the Eq. (8), the working profile of the indexing cam can be bulit though fitting all the cam curves. And then the cam will be obtained by adding the substrate and chamfer on the former working profile. In 
order to facilitate the analysis and design, the rollers and dial are fixed together rigidly. Based on the former design parameters, the mechanical elements of GIC model is assembled as figure 5 illustrated. (a)

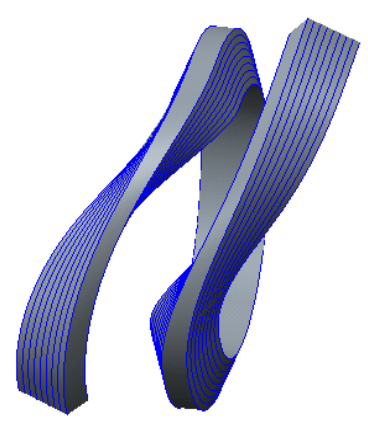

(b)

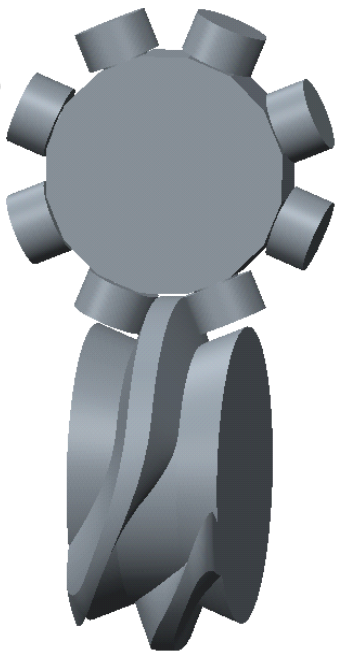

Figure 5. Virtual prototype of globoidal indexing cam: (a) the working profile of indexing cam; (b) the assembly model of GIC

\subsection{The kinematics simulation of virtual prototype}

The purpose of the kinematics simulation is to check the interference, evaluate the velocity and acceleration characteristics by examining relative movements between the mechanical parts.

After completing the assembly, the virtual prototype, which is exported and saved as form .x_t, is imported into the ADAMS software. Based on the working principle, the following constraint should be added to insure the correct operation: (1) Revolute pairs $\mathrm{R}_{1}$ between indexing cam and the rack, which allow the cam turning around its own axis. (2) Revolute pairs $\mathrm{R}_{2}$ between dial and the rack, which allow the dial turning around its own axis. (3) Contact pairs $\mathrm{C}$ between the indexing cam and the dial, which deliver movement and force. The virtual prototype is shown as Figure 6.

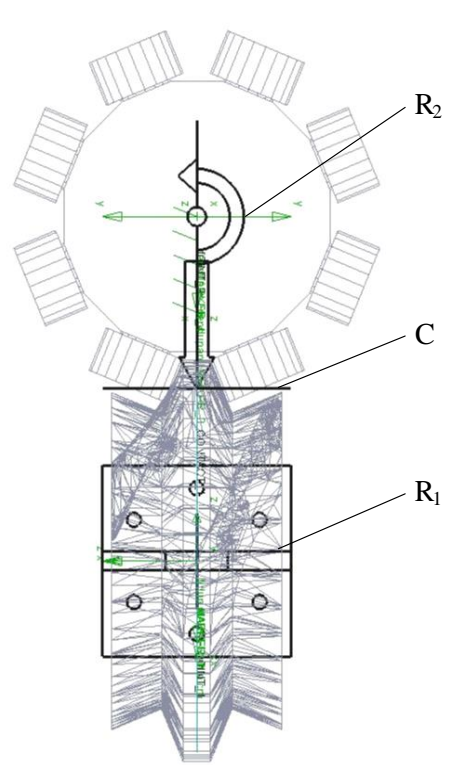

Figure 6. Virtual prototype of kinematics simulation

The indexing cam is driven by a constant speed motor, and the angular velocity is $\omega=\frac{\pi}{6} \mathrm{rad} / \mathrm{min}$, the simulation time is $t=24 \mathrm{~s}$, the step is $\mathrm{S}=5000$ (it's better to choose the steps as fewer as possible under the permitting conditions to get the more accurate result), and then the angular velocity curve of the dial can be obtained as Figure 7 shown.

Because of the vibration and impact, the two curves will nonlinear change around its own averaged value. The simulation curve of angular velocity show that the curve changes according to the sinusoid arcs periodically which is consistent with the design. Therefore, the virtual prototype and its theory of solving the working curvilinear equation in part 2 are approved to be correct and feasible by the kinematic simulation experiment based on the ADAMS curve data in Figure 7.

Angular velocity of dial

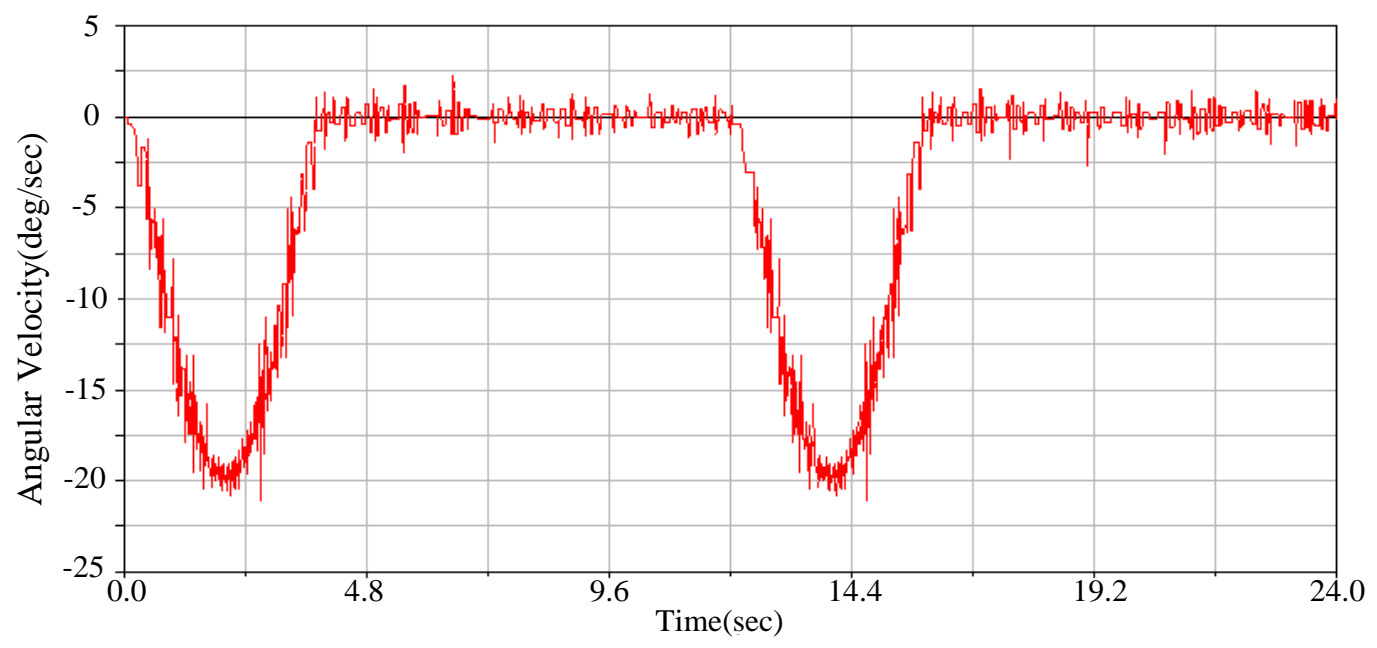

Fig 7. The angular velocity curve of dial 


\section{Conclusions}

(1) The working curvilinear equation of the indexing cam is obtained based on the RPY coordinate transformation method.

(2) According to the relative position of GIC components and the working curvilinear equation, virtual model is established by Creo2.0 software.

(3) The kinematics model was created by the ADAMS software and the kinematics simulation was carried out, which demonstrate the correctness and feasibility of the curvilinear equation established by the RPY method.

The method of creating the working curvilinear equation and the kinematics simulation data provide some reference for design and manufacture, and it has a certain significance for improving the cam quality.

\section{References}

1. HE Wei, PENG Guoxun, HU Yaping, CAO Weiqing. Chin. J. Mech. Engrg, 36, 33-38 (2000).

2. Giovanni Incerti. Proc. IEEE Int. Control Appl, 1392-1397 (2007).

3. Neklutin C. N. Mech. Cams Auto. Mach, (1969).

4. CHEN Fan Y. Mechanics. Des. Cam Mech, (1982).

5. Faydor L. Litvin, Ignacio Gonzalez-Perez, Kenji Yukishima, Alfonso Fuentes. Comput. Methods Appl. Mech. Engrg, 196, 4321-4336 (2007)

6. GE Rongyu, LI Taoyuan. J. Mac. Des, 28, 63-65 (2011).

7. Lin Wang, Yin Mingfu. Appl. Mech. Mater, 599, 517-521 (2014).

8. Yan H.S., Tsai M.C., Hsu M.H. Mec. Mac. Theory, 31, 397-412 (1996).

9. Reeve. J. Cams for Ind, (1995).

10. Li Lei, Zhang Chengliang, Wang Ming, Appl. Mech. Mater, 601, 581-586 (2014).

11. Jao-Hwa Kuang, Ah Der Lin, Tzong-Yow Ho. J. Mech. Des, 126, 909-915 (2004). 